\title{
Essential Thrombocytosis and Fatal Coronary Graft Occlusion: A Case Report
}

\author{
Joseph L. Blidgen, Clarence D. McGaw \\ Department of Surgery, Radiology, Anesthesia and Intensive Care, University of the West Indies, Mona P.O., Kingston, Jamaica \\ Email:josey702001@yahoo.com
}

How to cite this paper: Blidgen, J.L. and McGaw, C.D. (2020) Essential Thrombocytosis and Fatal Coronary Graft Occlusion: A Case Report. World Journal of Cardiovascular Surgery, 10, 187-191. https://doi.org/10.4236/wjcs.2020.1010021

Received: October 15, 2019

Accepted: October 23, 2020

Published: October 26, 2020

Copyright ( 2020 by author(s) and Scientific Research Publishing Inc. This work is licensed under the Creative Commons Attribution International License (CC BY 4.0).

http://creativecommons.org/licenses/by/4.0/

\begin{abstract}
Essential thrombocytosis (ET) is a rare myeloproliferative disorder, which is a major risk factor for thrombosis and bleeding, which complicates cardiovascular surgery. ET is a risk factor for coronary artery disease that should be treated. We present a case of a male with ET who underwent coronary artery bypass grafting (CABG) at the University Hospital of the West Indies, and died post-surgery. Post mortem revealed early graft thrombosis. We believe that post-operative aspirin therapy maybe would have prevented this fatal outcome.
\end{abstract}

\section{Keywords}

Thrombocytosis, Graft Occlusion, Coronary Artery Bypass Grafting

\section{Introduction}

Coronary artery disease (CAD) exhibiting symptoms usually suggest an etiology of obstructive disease due to underlying atherosclerosis. However, CAD may be due to unusual causes and should prompt further investigations. Essential thrombocytosis (ET) is one of the unusual causes of angina pectoris and myocardial infarction. In addition, patients with ET can present with cerebrovascular disease and ischemic stroke as well. This demonstrates ET as a predisposing factor for vasculopathies. Despite the propensity of ET for arterial thrombosis, a combination with heavy cigarette smoking can have a significant role in development of CAD in patients.

ET is a myeloproliferative disorder with a sustained elevated platelet count of greater than $800 \times 10^{9} / \mathrm{L}$, which can result in serious complications of arterial and venous thrombosis as well as bleeding in patients undergoing cardiovascular surgery. These complications can arise during or immediately after cardiovascu- 
lar surgery. ET should be treated with aspirin and thus in patients undergoing coronary artery bypass grafting (CABG), aspirin therapy should continue because of the high-risk of thrombo-embolic events in the high-risk ET patients. We report the case of a patient with ET who underwent CABG and subsequent died as a result of early postoperative graft thrombosis and occlusion.

\section{Case Report}

A fifty-one-year-old male was referred to the cardiothoracic service at the University Hospital of the West Indies (UHWI), Kingston, Jamaica two weeks after he was diagnosed with a ST segment elevation myocardial infarction. His electrocardiogram (ECG) showed ST-segment elevations in leads II, III and aVF, T wave inversion in $\mathrm{V} 2-\mathrm{V} 4$, and a right bundle branch block (RBBB).

He denied any prior chronic medical illnesses but gave a history of a 16-pack-year of cigarette smoking. He had no heart failure symptoms. He was also found to have thrombocytosis (platelet count-911 $\times 10^{9} / \mathrm{L}$ on initial admission to the peripheral institution, increasing to a peak of $1386 \times 10^{9} / \mathrm{L}$ within the ensuing week. Treatment was commenced with clopidogrel and aspirin (anti-platelet agents), heparin (anticoagulation), nitrates, statin, beta-blocker and hydroxyurea. He was subsequently referred to the Cardiologist at UHWI and underwent coronary angiography which revealed an $80 \%$ stenosis of the ostium of the left main artery (LMA) and 90\% mid vessel occlusion of the left anterior descending (LAD) artery. No significant occlusion was seen in the right coronary artery. His echocardiogram revealed mild left ventricular (LV) anteroseptal hypokinesia, mild right ventricular (RV) inferior wall hypokinesia and overall preservation of LV and RV function. Based on the coronary angiogram findings, a decision was made with the patient for immediate CABG. Of note, his immediate pre-operative platelet count had decreased to $650 \times 10^{9} / \mathrm{L}$.

The patient underwent $\mathrm{CABG} \times 2$ : left internal mammary artery (IMA) to the left anterior descending artery (LAD) and reverse saphenous vein graft to the obtuse marginal 1 (OM 1). The intraoperative course was uneventful. His activated clotting time (ACT) however, remained above the baseline (ACT: 273 seconds) and remained so, up to 15 minutes later, even after additional protamine. In view of the need to avoid excessive procoagulability in the postoperative period and as adequate hemostasis was achieved at this level of ACT, no further protamine was administered. At approximately 2 hours after surgery, the patient suddenly began complaining of chest pain, became diaphoretic and tachycardic and then had a cardiac arrest. Cardiopulmonary resuscitation was promptly instituted and the chest re-opened. There was no evidence of cardiac tamponade, no cardiac activity and the grafts were filled with clots. Despite all resuscitative measures the patient died.

Significant findings at postmortem were coronary artery occlusion, grafts with clots, recent myocardial infarction, and moderate atherosclerosis involving the renal and coronary arteries. The clinical impression was that the patient devel- 
oped acute occlusion of his coronary artery bypass grafts related to thrombocytosis and had a cardiac arrest.

\section{Discussion}

Symptomatic CAD is not always caused by a fixed atheromatous plaque, especially in young patients. ET is a rare condition and the incidence of CAD in ET is 9.4\% [1]. While the exact definition of thrombocytosis varies in the literature, a platelet count of $\geq 450 \times 10^{9} / \mathrm{L}$ is generally accepted value [2]. It may either be due to a reactive process (secondary thrombocytosis) or be caused by a clonal bone marrow disorder of unknown etiology. The distinction between the two is important as it carries implications for evaluation, prognosis and treatment. Clonal thrombocytosis associated with the myeloproliferative neoplasms, especially essential thrombocythemia and polycythemia vera, involves a multipotent hematopoietic progenitor cell and is manifested clinically by the overproduction of platelets in the absence of a definable cause. ET affects 2.5/100,000 persons/year [3]. About $9 \%$ to $22 \%$ of the patients with essential thrombocytosis present with vascular events (venous and/or arterial events) [3]. Thrombotic occlusions of large arteries are the main complications in ET; most commonly involving cerebral or coronary vessels. Thrombosis is a multifactorial disease in which the presence of a major risk factor should always be viewed as part of a complex interplay with other, less evident, factors [4].

While the mechanism of thrombosis remains elusive, thrombocyte dysfunction and increased number of platelets and activated granulocytes are known to play a role. Activated granulocytes are known to play an important role in platelet activation and endothelial injury and patients with ET have evidence of increased granulocyte activation as compared to normal controls [5].

While there are several established risk factors for CAD, the only confirmed risk factors in our case were that of chronic smoking and perhaps thrombo-embolism secondary to ET. We proffer that the peri-operative complications are highly likely due to the presence of ET. Despite ET causing arterial and venous thrombosis, equally important is the role that chronic cigarette smoking played in relation to platelets function in terms of aggregation but also platelet quality as well. Infact smoking maybe associated with accelerated erythropoiesis and thrombocytosis which are also hallmarks in ET and early stages of myelofibrosis by activation of certain signaling pathways [6].

Our patient's preoperative medications included hydroxyurea and the antiplatelet agents, aspirin and clopidogrel. This combination is the standard of care for patient with ET both preoperatively and postoperatively in CABG or percutaneous coronary intervention (PCI). From a surgical point of view, the patients with ET are apt to suffer complications such as thromboembolism or hemorrhage during the operation or postoperatively and hence it is desirable that the platelet count be reduced or normalized prior to surgery as a reduction of platelet count is often associated with improvement of outcomes [7]. This treatment 
resulted in reduction in the platelet count in our patient and there was no clinical evidence of ongoing symptomatic thrombotic events preoperatively. Studies have demonstrated a reduction of thrombotic events when aspirin is administered to patients with ET [8]. However, this occurred without a concomitant increase in the risk of hemorrhage. Beer, et al. recommend aspirin for all ET patients unless contraindicated [9]. Ruggeri et al. retrospectively studied the post-surgical outcomes in patients with polycythemia vera and essential thrombocythemia. Antiplatelet drugs were the optimal choice for anti-thrombotic prophylaxis in comparison with unfractionated heparin or low molecular weight heparin in patients with essential thrombocytosis. There was no correlation between bleeding episodes and the use of antiplatelet agents [10].

Cytoreductive therapy is recommended for high-risk patients. Hydroxyurea is the only cytoreductive agent proven to reduce thrombotic events in a randomized control trial [11]. Our patient was considered a high-risk patient with an elevated platelet count and vascular complications hence we commenced platelet reductive therapy.

The discussion about conduit choice in relation to vein or arterial conduits and risks of early venous graft failure should be considered. In this case, we went ahead with our usual conduit choice of LIMA and saphenous vein graft, bearing in mind the risk of early vein thrombosis and failure in this patient.

In view of the underlying pro-thrombotic phenotype of ET, the risks accompanying the hypercoagulable state associated with the stress of surgery are also magnified. This makes the perioperative management of patients with ET undergoing CABG surgery very challenging. There is an attempt by surgeons to balance the risk of thrombo-embolism with that of peri-operative hemorrhage. However, the fear of early surgical-specific bleeding and tamponade in our patient resulted in the delay of administration of aspirin post-operatively. We believe this resulted in acute graft occlusion.

\section{Conclusion}

ET is a rare but major risk factor for CAD and provides challenges in the peri-operative period. The thromboembolic risks are significant and it is recommended that continuous aspirin be administered in these patients.

\section{Conflict of Interest}

The authors have no conflicts of interest related to the publication of this manuscript.

\section{References}

[1] Kumagai, N., Mitsutake, R., Miura, S., Kawamura, A., Takamiya, Y., Nishikawa, H., et al. (2009) Acute Coronary Syndrome Associated with Essential Thrombocythemia. Journal of Cardiology, 54, 485-489. https://doi.org/10.1016/j.jjcc.2009.03.001

[2] Skoda, R.C. (2009) Thrombocytosis. Hematology Am Soc Hematol Education Pro- 
gram, 2009, 159-167. https://doi.org/10.1182/asheducation-2009.1.159

[3] Hehlmann, R., Jahn, M., Baumann, B. and Kopcke, W. (1988) Essential Thrombocythemia. Clinical Characteristics and Course of 61 Cases. Cancer, 61, 2487-2496. https://doi.org/10.1002/1097-0142(19880615)61:12<2487::AID-CNCR2820611217> 3.0.CO;2-T

[4] Landolfi, R., Cipriani, M.C. and Novarese, L. (2006) Thrombosis and Bleeding in Polycythemia Vera and Essential Thrombocythemia: Pathogenetic Mechanisms and Prevention. Best Practice \& Research Clinical Haematology, 19, 617-633. https://doi.org/10.1016/j.beha.2005.07.011

[5] Falanga, A., Marchetti, M., Vignoli, A., Balducci, D. and Barbui, T. (2005) Leukocyte-Platelet Interaction in Patients with Essential Thrombocythemia and Polycythemia Vera. Experimental Hematology, 33, 523-530.

https://doi.org/10.1016/j.exphem.2005.01.015

[6] Hasselbalch, H.C. (2015) Smoking as a Contributing Factor for Development of Polycythemia Vera and Related Neoplasms. Leukemia Research, 39, 1137-1145. https://doi.org/10.1016/j.leukres.2015.09.002

[7] Gunz, F.W. (1960) Hemorrhagic Thrombocythemia: A Critical Review. Blood, 15, 706-723. https://doi.org/10.1182/blood.V15.5.706.706

[8] Michiels, J.J. (1999) Aspirin and Platelet-Lowering Agents for the Prevention of Vascular Complications in Essential Thrombocythemia. Clinical and Applied Thrombosis/Hemostasis, 5, 247-251. https://doi.org/10.1177/107602969900500408

[9] Beer, P.A., Erber, W.N., Campbell, P.J. and Green, A.R. (2011) How I Treat Essential Thrombocythemia. Blood, 117, 1472-1482.

https://doi.org/10.1182/blood-2010-08-270033

[10] Ruggeri, M., Rodeghiero, F., Tosetto, A., Castaman, G., Scognamiglio, F., Finazzi, G., et al. (2008) Postsurgery Outcomes in Patients with Polycythemia Vera and Essential Thrombocythemia: A Retrospective Survey. Blood, 111, 666-671. https://doi.org/10.1182/blood-2007-07-102665

[11] Cortelazzo, S., Finazzi, G., Ruggeri, M., Vestri, O., Galli, M., Rodeghiero, F., et al. (1995) Hydroxyurea for Patients with Essential Thrombocythemia and a High Risk of Thrombosis. The New England Journal of Medicine, 332, 1132-1136. https://doi.org/10.1056/NEJM199504273321704 